\title{
Inflammatory Myofibrohistiocytic Proliferation Presenting as Giant Gastric Pseudotumor
}

\author{
Charles S. Marn and Frank K. Hsu \\ Department of Radiology, University of Michigan Medical Center, Ann Arbor, Michigan, USA
}

\begin{abstract}
A 5-year-old boy presented with refractory microcytic anemia, growth failure, and markedly elevated sedimentation rate. Computed tomographic (CT) and upper gastrointestinal study disclosed an ulcerated 9-cm large gastric mass, which proved to be an inflammatory myofibrohistiocytic proliferation (IMP). The characteristic clinical and radiologic features of this rare entity are herein reviewed.
\end{abstract}

Key words: Stomach, neoplasms-Inflammatory pseudotumor, diagnosis.

The term inflammatory myofibrohistiocytic proliferation (IMP) has been recently coined to encompass an array of benign soft tissue lesions previously designated as plasma cell granuloma, granuloblastoma, inflammatory pseudotumor, fibrohistiocytoma, inflammatory fibroid polyp, lymphoid hamartoma, eosinophilic granuloma, and xanthomatous pseudotumor $[1,2]$. This lesion is a benign proliferation of myofibroblasts and inflammatory cells, and is most often found in the lungs of young adults $[1,3]$. Intraabdominal involvement by IMP can occur with lesions involving the wall of the stomach, duodenum and ileum, or the mesentery or retroperitoneum [16].

While this case constitutes only the fifth reported example of gastric IMP in children [1, 3, 7], this collective experience is noteworthy for two trends. First, a typical set of clinical, laboratory, and imaging features is emerging as the hallmark of this disorder. Second, the reported cases are recent, raising

Address offprint requests to: Charles S. Marn, M.D., Department of Radiology, Box 0030, University of Michigan Hospitals, 1500 East Medical Center Drive, Ann Arbor, MI 48109-0030, USA the possibility that gastric IMP in children may have been misclassified as sarcoma in the past.

\section{Case Report}

A 5-year-old boy was evaluated because of persistent growth retardation, pallor, and fatigue. Past medical history was notable only for a hospital admission for ethanol ingestion at the age of 30 months. Initial outpatient evaluation revealed severe iron deficiency anemia (hemoglobin $1.6 \mathrm{~g} / \mathrm{dl}$ ), but no fever, chills, bruising, hematuria, or melena. The child was transfused to a hemoglobin of $8 \mathrm{~g} / \mathrm{dl}$, but quickly fell back to a hemoglobin of $3 \mathrm{~g} / \mathrm{dl}$ while on iron drops. An intravenous urogram demonstrated a left-sided mass, perhaps arising from the left kidney. He was transferred to our institution for further evaluation. Laboratory tests confirmed the iron deficiency anemia (hemoglobin $3.9 \mathrm{~g} / \mathrm{dl}$, MCV $65 \mathrm{fl}$, serum iron $<20 \mu \mathrm{g} / \mathrm{dl}$ ), and demonstrated marked elevation of the erythrocyte sedimentation rate at greater than $150 \mathrm{~mm} / \mathrm{h}$. Physical exam was remarkable for severe growth retardation, with both height and weight $(92 \mathrm{~cm}$ and $13.6 \mathrm{~kg}$, respectively) significantly below the fifth percentile and bone age being about 3 years.

Computed tomography (CT) demonstrated an intragastric mass with a central collection of contrast (Fig. 1). No liver lesions or enlarged lymph nodes were evident, and the kidneys and adrenal glands were normal. An upper gastrointestinal examination confirmed the ulcerated giant mass (Fig. 2), and a differential diagnosis of leiomyoma, sarcoma, and lymphoma was offered.

Upper endoscopy demonstrated a large, lobulated friable mass, and its biopsy suggested IMP with no evidence of malignancy. Given the patient's symptoms, however, a partial gastrectomy was performed.

Histologic examination of the resected $9 \mathrm{~cm}$ mass with ulceration showed cellular proliferation extending to the serosa. Strands of plasma cells were interposed between layers of myofibroblasts with a dense collagenized background. Immunoperoxidase stains revealed that the plasma cell infiltrate was polyclonal.

\section{Discussion}

IMP is the current designation for a poorly understood entity consisting of mass-like accumulation of myofibroblasts and inflammatory cells [1]. The le- 

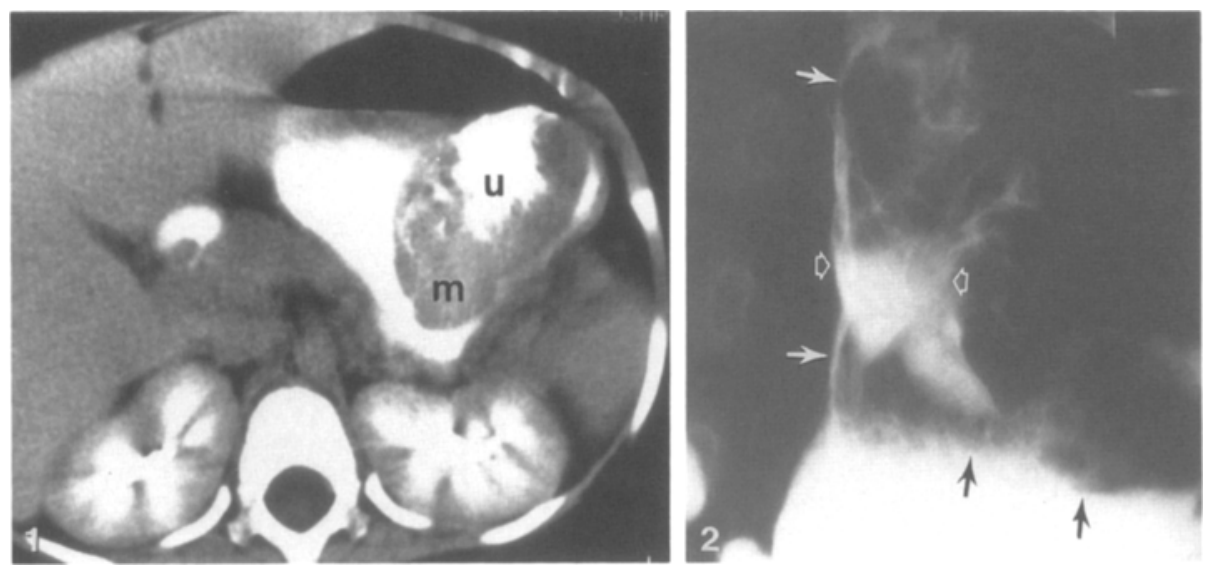

Fig. 1. CT scan with oral and intravenous contrast. A large, endophytic gastric mass $(m)$ is present with a central, irregular ulceration $(u)$.

Fig. 2. Upper gastrointestinal examination. A lobulated gastric mass (closed arrows) protrudes into the lumen. An irregular central ulcer (open arrows) is noted.

sion is most common in the lung, but can occur in the stomach, small intestine, mesentery, liver, pancreas, kidney, retroperitoneal space, lymph nodes, and even the brain and meninges [1-6]. It is uncertain whether the lesion is neoplastic or inflammatory in nature. In some cases, an inciting event, such as surgery, trauma, infection, or malignancy, is present as a precipitating factor, supporting the claim that this lesion represents an exaggerated response to injury $[3-5,7]$. A suspected role for altered immunity is supported by the observation that some cases are associated with other unusual inflammatory lesions, such as sclerosing cholangitis, and retroperitoneal fibrosis [3]. In our case, ethanol ingestion requiring hospitalization occurred 3 years before presentation with IMP, but the extent of that gastric injury, if any, was not documented. The benign nature of the lesion is supported by the lack of distant metastatic disease, and little propensity for recurrency $[1,3,5,8]$.

A constellation of typical clinical features, if recognized, suggests the correct diagnosis. Severe microcytic anemia refractory to iron therapy is common $[1,3,9,10]$. This occurs in IMP in any anatomic location and is not associated with gastrointestinal blood loss. Many cases exhibit growth failure $[1,3$, 9]. Depressed somatomedin levels, which normalized after the removal of a retroperitoneal IMP, were documented in one case with iron deficiency anemia and growth failure, but the relationship of isolated hormonal deficiency with the clinical symptoms is unclear [9]. The sedimentation rate is often markedly elevated $[1,5,9]$. Elevated serum immunoglobulins are frequently seen, perhaps produced directly by the plasma cell infiltrate. All of these abnormalities are reversed by surgical removal of the mass. Iron deficiency anemia, growth failure, and elevated sedimentation rate were present in our case.

The detection of a bulky gastric wall mass in a child usually indicates a malignant process. Primary gastric wall tumors include lymphoma and various sarcomas, while direct extension of other intraabdominal neoplasms, such as Wilm's tumor or neuroblastoma, may involve the stomach wall. The CT scans in this case proved the lesion to have originated within the gastric wall, and thus limited the preoperative differential diagnosis to the gastric malignancies of childhood. Endoscopic biopsy was the first test to suggest the correct diagnosis, and IMP singularly explains the patient's entire clinical picture.

Surgery is required for confirmation of the diagnosis and relief of symptoms. Patients typically have a rapid resolution of the anemia, and a growth spurt often occurs in the year following resection $[1,3,9]$. The benign nature of the lesion dictates surgical management. Complete excision with the least radical surgical procedure should be performed [8]. Recurrence is rare and is usually successfully managed with a second resection. The potential to misclassify this lesion as a sarcoma does exist $[1,8]$, and a coordinated effort between the surgeon, radiologist, pediatrician, and pathologist is necessary for correct diagnosis and therapy.

\section{References}

1. Tang TT, Segura AD, Oechler HW, Harb JM, Adair SE, Gregg DC, Camitta BM, Franciosi RA. Inflammatory myofibrohistiocytic proliferation simulating sarcoma in children. Cancer 1990;65:1626-1634

2. Bolck F, Katenkamp D. Granuloblastomas of the stomach (so-called eosinophilic granulomas) - a variant of fibrous histocytomas? Pathol Res Pract 1981;171:336-344

3. Maves CK, Johnson JF, Bove K, Malott RL. Gastric inflammatory pseudotumor in children. Radiology 1989;173:381383

4. Soga J, Saito K, Suzuki N, Sakai T. Plasma cell granuloma of the stomach: a report of a case and review of the literature. Cancer 1970;25:618-625 
5. Day DL, Sane S. Dehner LP. Inflammatory pseudotumor of the mesentery and small intestine. Pediatr Radiol $1986 ; 16: 210-215$

6. Neerhout RC, Larson W, Mansur P. Mesenteric lymphoid hamartoma associated with chronic hypoferremia, anemia, growth failure and hyperglobulinemia. New Engl $\mathrm{J}$ Med $1969 ; 17: 922-925$

7. Gaillard D, Daoud S, Tholon JP, Lallemand A. Inflammatory pseudotumor (IPT) of the stomach. Pediatr Pathol 1987;7:486

8. Freud E, Bilik R, Yaniv I, Horev G, Cohen D, Mimouni M, Zer M. Inflammatory pseudotumor in childhood. Arch Surg 1991;126:653-655
9. Buchanan GR, Chipman JJ, Hamilton BL, Daughaday WH. Angiomatous lymphoid hamartoma: inhibitory effects on erythropoiesis, growth, and primary hemostasis. $J$ Pediatr 1981;99:382-388

10. Boxer LA, Boxer GJ, Flair RC, Engstrom PF, Brown GS. Angiomatous lymphoid hamartoma associated with chronic anemia, hypoferremia, and hypergammaglobulinemia. $J P e$ diatr 1972;81:66-70

Received: January 7, 1992; accepted: February 12, 1992 